\title{
Sarcoidosis of the hypothalamus and pituitary stalk
}

\author{
Thomas J. Vogl ${ }^{1}$, Joachim Stemmler ${ }^{1}$, Peter C. Scriba ${ }^{2}$, Claudia Vogl ${ }^{2}$, and Josef Lissner ${ }^{1}$ \\ ${ }^{1}$ Department of Radiology, University of Munich, Ziemssenstraße 1, W-8000 Munich 2, FRG \\ ${ }^{2}$ Department of Internal Medicine, University of Munich, Munich, FRG
}

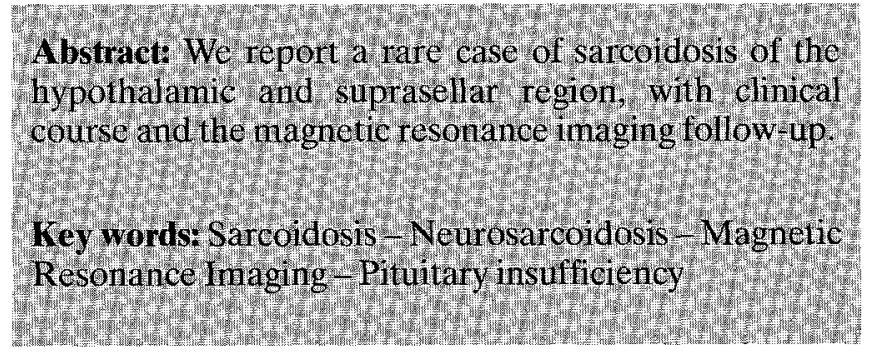

\section{Introduction}

Sarcoidosis is a multisystem, noncaseating, granulomatous disease of unknown origin [1]. Involvement of the central nervous system has been observed in up to $14 \%$ of autopsies in cases of systemic sarcoidosis [2].

We present the case of a 52-year-old man with a sarcoid granuloma in the hypothalamus and suprasellar region, detected with magnetic resonance imaging (MRI). The lesion was followed up by two further MR examinations, while the patient was treated with high-dose corticosteroids and hormone replacement.

\section{Case report}

The patient had suffered only ever sports injuries. In July 1989, he had an attack of extreme pain in the left flank and high fever after an exhausting tour in the mountains. Renal calculi were diagnosed. In September 1989, he felt increasing chills and fatigue during a sailing tour and, 1 month later, had an attack of fever $\left(39^{\circ} \mathrm{C}\right)$, chills without respiratory infection, weakness and fatigue. On $13 \mathrm{March}$ 1990 , a chest radiograph showed bilateral hilar lymph node enlargement. Erythema nodosum was observed at the same time, leading to the diagnosis of sarcoidosis. The patient then recalled that he had had pain in both ankles early in 1989. In April 1989, the patient had rapidly developed diabetes insipidus with a maximum of 101 fluid intake per day. The diabetes insipidus was aggravated

Offprint requests to: T.J. Vogl when prednisolone was given as treatment for sarcoidosis. At the same time, severe hypotension was detected.

Suspected hypopituitarism was confirmed by cortisol levels below $4 \mu \mathrm{g} / \mathrm{dl}$ throughout the day ( 3 examinations) and a response to stimulation by ACTH $(32,5 \mu \mathrm{g} / \mathrm{dl})$. The patient immediately felt stronger when given prednisolone. He had a low basal TSH $(0,2 \mathrm{mu} / \mathrm{l})$ with a poor response to stimulation by TRH (4,37 mu/l). In May 1990, perimetry showed a left upper outer quadrant field defect. These findings indicated a diagnosis of acute sarcoidosis with anterior and posterior pituitary insufficiency, secondary to sarcoidosis of the pituitary stalk and hypothalamus.

An MR examination in July 1990, when the patient was already receiving prednisolone, revealed on $\mathrm{T} 1$-weighted images a $21 \times 12 \times 15 \mathrm{~mm}$ lesion in the hypothalamus and suprasellar region (Fig. 1). The lesion was of variable signal intensity both before and after i.v. Gd-DTPA application. The hypothalamic portion was uniformly hyperintense, but the presumed cystic suprasellar portion was isointense with grey matter before injection and showed marginal enhancement. This component extended up beyond the optic chiasm, explaining the visual defect.

Corticosteroid therapy was continued and, in September 1990, a follow-up showed a minor reduction in size of the hypothalamic portion of the lesion (Fig. 2). The suprasellar portion was unchanged in size; neither showed any change in signal intensity with Gd-DTPA.

The corticosteroids were gradually reduced to $10 \mathrm{mg} / \mathrm{d}$ by December 1990. The visual defect remained unchanged and a second follow-up MR examination in November 1990 (Fig. 3) showed a slight increase in size of the hypothalamic portion, which was now isointense with grey matter on T1-weighted images and showed only minor enhancement. The suprasellar part was unchanged in size and signal intensity. The patient developed a complete temporal hemianopia in the left eye during summer 1990, while the right eye showed an upper outer quadrant defect, which remained unchanged. The patient declined surgery for these 

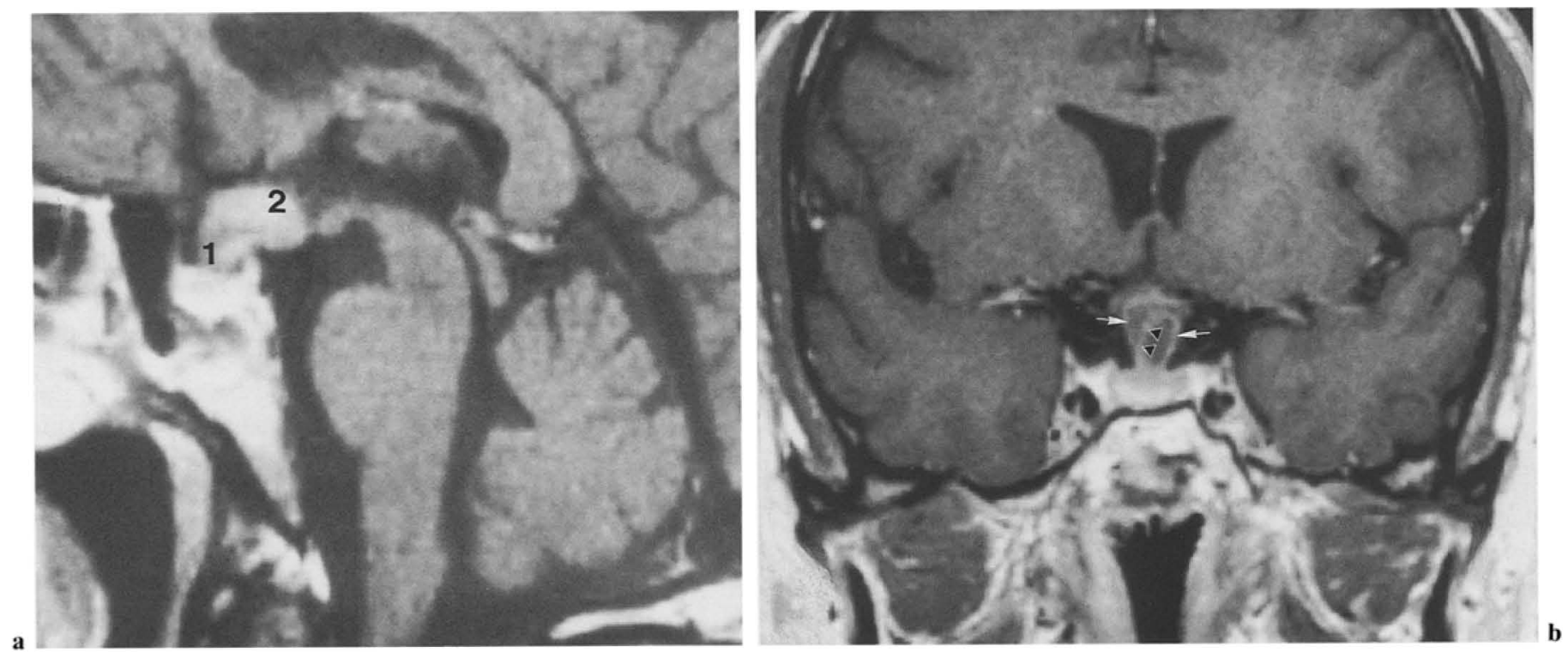

Fig.1a-c. MRI July 1990. T1-weighted sagittal (a) (TR=600, TE $=35 \mathrm{~ms}$ ) after Gd-DTPA demonstrates a uniformly hyperintense lesion with suprasellar $(I)$ and hypothalamic components (2). (b) T1-weighted coronal image ( $\mathrm{TR}=500$,

$\mathrm{TE}=15 \mathrm{~ms}$ ) after Gd-DTPA demonstrates the cystic suprasellar portion to show marginal enhancement (arrows); pituitary stalk (arrowheads). Note the relationship of the lesion to the optic chiasm and the pituitary gland. (c) T1-weighted coronal, unenhanced image, more posterior, shows the granulomatous tissue in the hypothalamus to be of spontaneously high signal intensity.

c

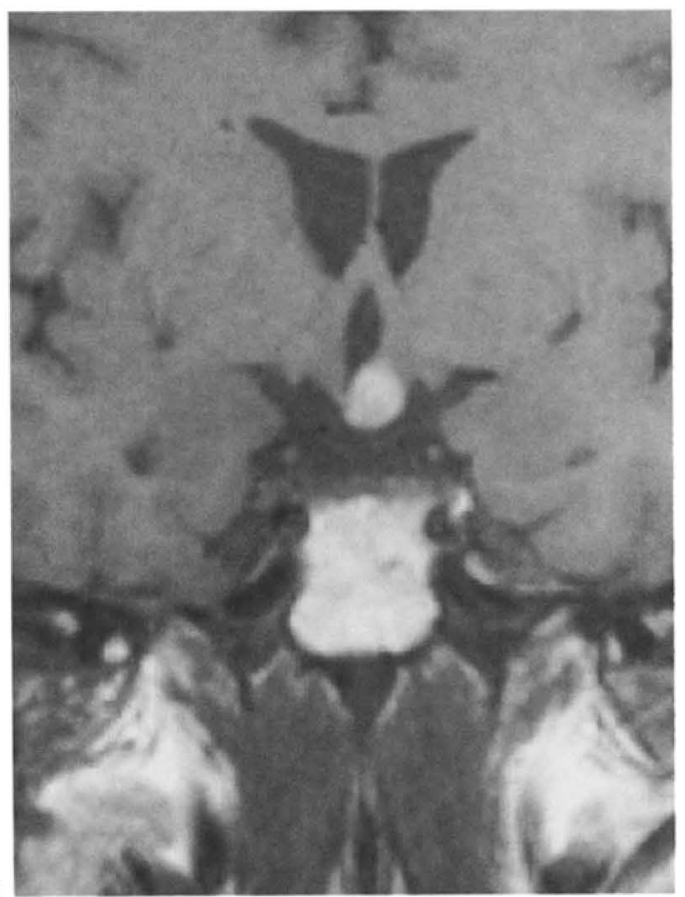

Fig.2. Sagittal T1-weighted image in September 1990 demonstrates a decrease in size of the hyperintense hypothalamic component, while the presumed cystic suprasellar portion is unchanged.

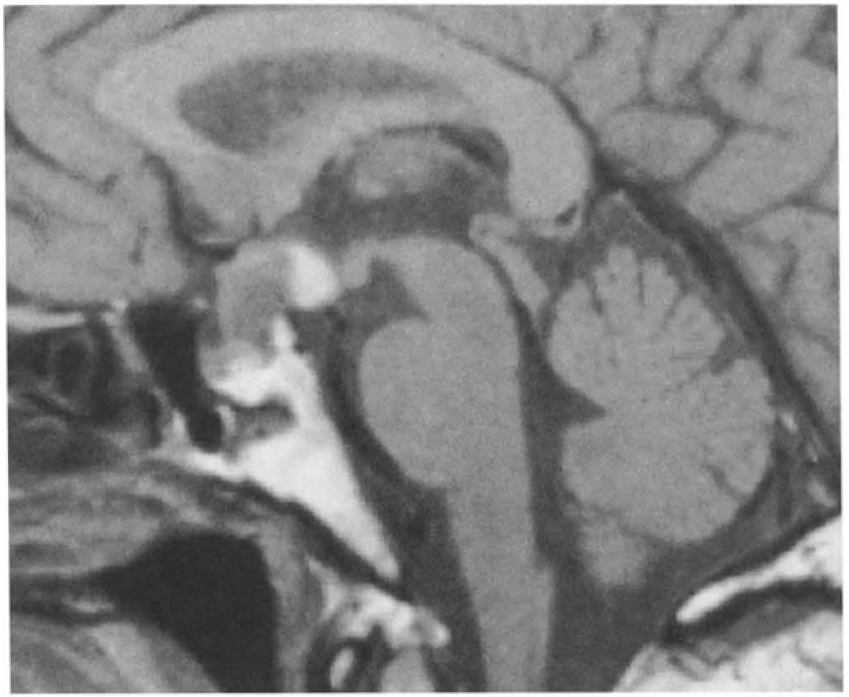

defects at this time. He went back to work on replacement therapy with thyroxine, testosterone and DDAVP. Prednisolone, started with $80 \mathrm{mg} / \mathrm{d}$, was gradually reduced to $10 \mathrm{mg} / \mathrm{d}$ (December 1990).

Due to the increasing visual defect the patient agreed to surgical therapy in May 1991. The intraoperative findings showed a cystic suprasellar portion and a homogeneous component in the hypothalamus. The microscopical appearance of the surgical specimen tends to verify the diagnosis of sarcoidosis.

\section{Discussion}

Clinical involvement of the central nervous system (CNS) is seen in approximately $5 \%$ of cases of systemic sarcoidosis and may be the initial presentation in $2.5 \%$ [3]. In- 

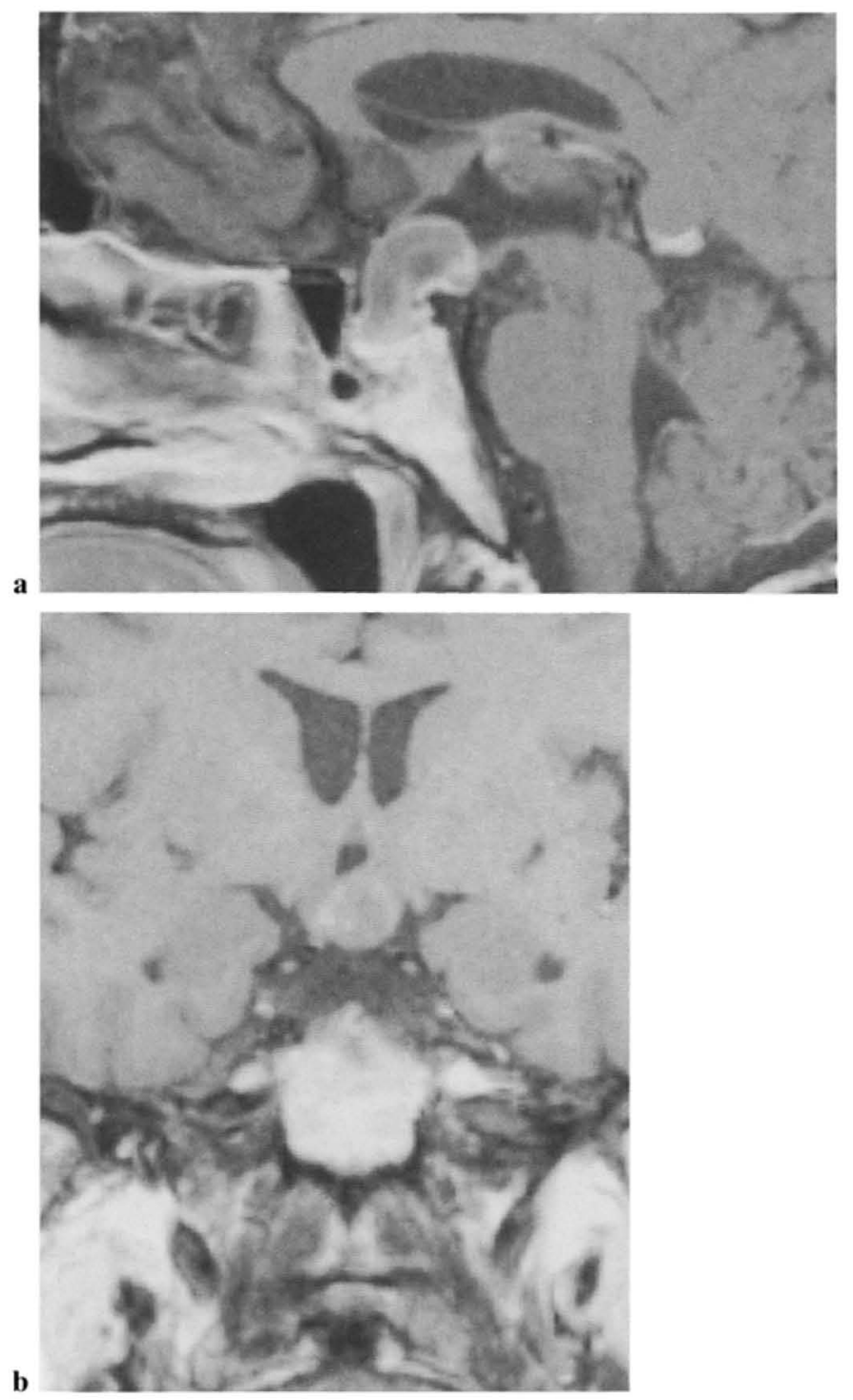

Fig. 3a and b. Sagittal (a) and coronal (b) image after Gd-DTPA in November show alterations in signal intensity and size in both parts of the lesion; the presumed cystic component being more extensive.

volvement of the hypothalamic-pituitary region with resulting anterior pituitary insufficiency is even rarer, with 51 cases reported up to 1989 [4]. CNS manifestations of sarcoidosis include cranial neuropathies due to compression or infiltration in $50-70 \%$ of affected cases. The most frequent manifestation (up to $50 \%$ ) is a peripheral seventh nerve palsy. Meningeal infiltration, leading to aseptic meningitis, is seen in approximately $20 \%$, hydrocephalus due to ventricular outlet obstruction in $15 \%$ and local parenchyma lesions with resulting hypophysealhypothalamic dysfunction in $15-25 \%[3,5]$.

Contrast-enhanced C $T$ is helpful in the investigation of suspected intracranial sarcoid granulomas $[3,5,6]$, although the findings are not specific and may resemble those at meningiomas, lymphoma and primary or metastatic tumours $[8,9]$. CT is of limited value for detection of lesions in the hypothalamus and the hypophyseal region. In our case, enhanced CT did not show the hypothalamic lesion and gave insufficient detail to assess the size of the suprasellar portion. If involvement of this region is suspected, MR is indicated.

MR provided greater sensitivity than $\mathrm{CT}$ in the assessment of hypothalamic-hypophyseal involvement, although there is a great variability in the appearances of intracranial sarcoidosis on MR [7]. The signal may be iso- or hypointense compared to grey matter on T1-weighted images $[7,10]$.

These signal characteristics do not help to differentiate these lesions from en plaque meningiomas, lymphoma and unusual metastases [8,9]. Especially the craniopharyngioma with cystic and solid components is an important differential diagnosis in the suprasellar region. The MR appearance of the craniopharyngioma is variable. Solid portions of this lesion tend to be isointense to grey matter, whereas cystic components tend to be hyperintense on T1weighted images [11]. The first MR examination in our case showed the signal intensities of the two components of the lesion to be different and they changed in the follow-up examinations; both showed lower signal intensity before and after Gd-DTPA administration on the last examination.

There was good correlation of clinical and MR findings. The patient developed diabetes insipidus which could be explained by the hypothalamic lesion. The suprasellar portion of the granuloma extended above the optic chiasm and infiltrated the pituitary stalk, explaining anterior pituitary insufficiency, with low cortisol and TSH levels, and the visual defect.

MRI is most helpful for detection of sarcoid involvement of the hypothalamus and pituitary region and is the technique of choice for the follow-up and assessing the response to steroid therapy.

\section{References}

1. Wiederholt WC, Siekert RG (1965) Neurological manifestations of sarcoidosis. Neurology 15: 1147-1154

2. Ricker W, Clark M (1962) Sarcoidosis: a clinic pathology review of 300 cases, including 22 autopies. Am JCPathol 91: 625-749

3. Stern BJ, Krumholz A, Johns C, Scott P, Nissim J (1985) Sarcoidosis and its neurological manifestations. Arch Neurol 42: 909 917

4. Veseley DL (1989) Hypothalamic sarcoidosis, a new cause of morbid. South Med J 82: 758-761

5. Delaney P (1977) Neurological manifestations in sarcoidosis, review of the literature with a report of 23 cases. Ann Intern Med 87: 336-345

6. Kendall BE, Tatler GLV (1978) Radiological findings in neurosarcoidosis. Br J Radiol 51: 81-92

7. Hayes WS, Sherman JL, Stern BJ, Citrin CM, Pulaski PD (1987) MR and CT evaluation of intracranial sarcoidosis. AJNR 8: 841847

8. Brant-Zawadzki M, Norman D (1987) Magnetic resonance imaging of the central nervous system. New York: Raven Press

9. Spagnoli MV, Goldberg HI, Grossmann RI, et al. (1986) Intracranial meningiomas: High field MR imaging. Radiology 161: 369-375

10. Reed LD, Abbas S, Markivee CR, Fletcher JW (1986) Neurosarcoidosis responding to steroids. AJR 146: 819-821

11. Linden CN, Martinez CR, Gonzalvo AA, Cahill DW (1989) Intrinsic third ventricle craniopharyngioma: CT and MR findings J Comput Assist Tomogr 13: 362-368 